(2) OPEN ACCESS

\title{
Systematically capturing and acting on insights from front-line staff: the 'Bedside Learning Coordinator'
}

\author{
Jenny Shand (1) , ${ }^{1,2}$ Dominique Allwood, ${ }^{3,4}$ Nicole Lee, ${ }^{5}$ Noor Elahi, ${ }^{6}$ \\ Iain McHenry, ${ }^{7,8}$ Karen Chui, ${ }^{9}$ Sophie Tang, ${ }^{10}$ Zoe Dawson-Couper, ${ }^{11,12}$ \\ James Mountford, ${ }^{13}$ Richard Bohmer ${ }^{14}$
}

For numbered affiliations see end of article.

\section{Correspondence to} Dr Jenny Shand, UCLPartners, London W1T 7HA, UK; jenny.shand@uclpartners.com

Received 8 July 2020 Revised 11 January 2021 Accepted 13 January 2021 Published Online First 5 February 2021

\section{Check for updates}

(C) Author(s) (or their employer(s)) 2021. Re-use permitted under CC BY-NC. No commercial re-use. See rights and permissions. Published by BMJ.

To cite: Shand J, Allwood D, Lee N, et al. BMJ Qual Saf 2021;30:509-512.
Insights from front-line staff are generally agreed to be vital for informing quality improvement. However, health services often struggle to gather internal experiencebased insights from staff systematically. When such data are collected, standard, systematic mechanisms are often lacking to act on the insights the data convey. To better exploit this potentially rich source of insights we propose that health services invest in a systematic mechanism to gather data from front-line experience. We trialled one such mechanism, the 'Bedside Learning Coordinator' (BLC) at the National Health Service (NHS) Nightingale Hospital London (Nightingale), a field hospital established in an exhibition centre to provide additional ventilated bed capacity for London's patients with COVID-19. ${ }^{1}$

Front-line healthcare staff whose roles and experience give them rich insights and ideas across a range of dimensions (including how to improve patient care, workplace efficiency and staff well-being) often lack time or power to enact change or make systemic change. One study found that nurses solved most of the problems they encountered locally, escalating only $7 \%$ of problems up the organisation for definitive solutions to be designed and implemented. ${ }^{2}$ While local problem solving can be helpful, a default to firefighting and finding local 'workarounds' rather than systematic improvements mean that valuable systemic learning is lost and standard ways of working are not improved as part of routine operations. The net result may be worse organisational performancecombined with wasted resource and frustration for staff. ${ }^{3}$

In contrast, senior hospital decisionmakers can effect change, but may not have timely access to 'ground-level', rich staff and patient insights to inform changes. Qualitative insights from patients and front-line staff are seldom part of routine data capture, and when captured are frequently underexploited. Furthermore, failure to enact local operational change contributes to healthcare's slow pace of innovation adoption ${ }^{4}$ and can also lead to staff dissatisfaction and disengagement.

The role of the BLC was created with the aim of sourcing ideas for improvement, taking those insights to decision-makers who can act on them to improve standard work, and to communicate changes back to front-line staff and monitor success of implementation.

\section{LEARNING SYSTEMS}

Effective learning systems are characterised by their ability to both sense and respond. This requires a rich data stream from front-line operations and an ability to act on those insights. This includes escalation to the appropriate decision-makers, integration of information from internal and external sources to inform design of effective changes, reliable implementation into practice and feedback loops to ensure implemented changes are successful. This closed-loop structure and associated behaviours create the foundations for systematic learning. Although most healthcare delivery organisations can do most of those things, although not always joined up, the ability to capture insight from staff at the bedside and return change back to the bedside often remains challenging.

For Nightingale, the insight deficit was clear. ${ }^{1}$ There was uncertainty across both operational and clinical domains. Staff were redeployed from different NHS organisations; equipment, such as ventilators and anaesthetic machines, was sourced from 


\begin{tabular}{|c|c|}
\hline Gathering insights from the bedside & $\begin{array}{l}\text { Taking agreed system changes back to the } \\
\text { bedside }\end{array}$ \\
\hline 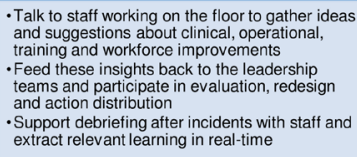 & 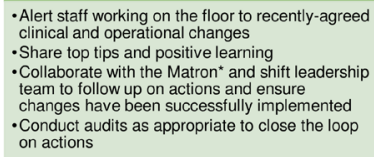 \\
\hline The BLC is there to... & The BLC cannot be relied upon to... \\
\hline 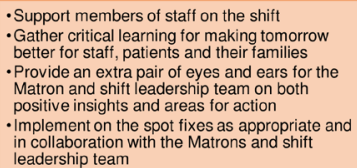 & $\begin{array}{l}\text { - Provide direct clinical care } \\
\text { - Directly lead the response to clinical critical } \\
\text { incidents } \\
\text { - Replace the role of the Matron or other shift } \\
\text { leaders the } \\
\text { - Fill rota" gaps in the event of staff absence }\end{array}$ \\
\hline
\end{tabular}

Figure 1 The role of the Bedside Learning Coordinator (BLC). *Matrons are senior nurse providing clinical leadership to the ward team alongside the lead consultant. ${ }^{* *} A$ rota is a system for planning the staffing for different duties for the ward over a set time period.

multiple suppliers and not standard; understanding of COVID-19 and its treatment was rapidly evolving. These factors contributed to the learning challenge and the resulting desire to implement a learning system with the ability to capture front-line insights for improvement in real-time and use them to inform action at its core.

\section{THE BLC ROLE}

BLCs were drawn from a range of professional backgrounds. A clinical background was not a prerequisite for the role in recognition that great ideas can come from outside of the conventional team. What was more important was observational skill, an interest in processes and systems, and commitment to the learning and improvement ethos. The role has parallels to well-described researcher-in-residence models, ${ }^{5}$ but was not a researcher or evaluator, rather a rostered member of the ward team. See figure 1 for a summary of the role.

Using a semistructured form, BLCs captured staff insights and observations about what was and was not working well. The form had six categories: staff wellbeing, workflow and processes, equipment, staff (skill mix), clinical protocol and other. They were encouraged to collaborate with staff, make changes where appropriate in real time and document these, and work alongside the matron (senior nurse providing clinical leadership to the ward team alongside the lead consultant). In addition to harvesting suggestions for improvement, BLCs also alerted staff to agreed changes, whether arising from external advice or internal redesign. They also undertook occasional focused audits to confirm that agreed changes were satisfactory to staff and implemented as planned, thereby helping to close the learning cycle.

\section{INSIGHTS CAPTURED FROM EXPERIENCE}

Research outputs are generally triaged according to the significance and strength of the findings. ${ }^{6}$ For experience-based data, there is not an equivalent triage system. Insights typically range from operational 'quick fixes' to areas for more formal evaluation.

At the Nightingale, insights were triaged into three areas (see table 1): 'Fix' (requiring action where it is well known how to manage the problem), 'Improve' (suggestions for better ways of doing things) and 'Change' (requiring substantial changes). BLCs worked within the small central Quality and Learning Team ${ }^{1}$ to triangulate observations from the bedside with other data sources, such as incident reports and performance dashboards, and to ensure support from and connection with senior leaders

\section{Table 1 Fix, Improve, Change: defining, triaging and addressing problems in the learning system

\begin{tabular}{lll}
\hline Category & Definition & Examples \\
\hline Fix & $\begin{array}{l}\text { Resolve problems in reliably doing what we said we would } \\
\text { do. These were usually issues that could be resolved with } \\
\text { rapid operational changes. }\end{array}$ & $\begin{array}{l}\text { Insting mirrors into the donning area to improve the safety and reliability of } \\
\text { staff. }\end{array}$
\end{tabular} \\ Ensuring contact telephone numbers were correct and prominently displayed on the ward. \\ Ensuring adequate stock levels and visible storage of drugs and key supplies. \\ Improve Find better ways of delivering standard care; improve what Introducing a day zero simulation training day ahead of individuals' first shifts is currently being done. to improve readiness of staff for the floor and clinical environment (staff came from different hospitals, clinics or organisations that had different ways of working and different equipment).}

Streamlining the donning process at shift handover: reduce the time for staff to put on PPE and ensure it is correctly worn before entering the clinical area.

Change Significant changes in clinical or operational practice. The design, development and implementation of a new venous thromboembolism protocol.

Developing the extubation protocol.

When a challenge arose, the Quality and Learning Team would define the problem by synthesis across the Bedside Learning Coordinator observations and insights and any additional relevant data sources, categorise and triage the problem. Next, the appropriate Specialist Decision Forum would review and either decide on a change to be implemented or pass along to the daily Clinical Forum (a multidisciplinary meeting) for a decision on a change or, if appropriate, to designate for simulation, testing and research. Source: Bohmer et al. ${ }^{1}$ 
when influencing action. The system recognised that some insights require more detailed critical and rigorous evaluation before putting them into practice. A tracker tool contained the live status of observations and actions and was used to both manage progress and inform staff of changes.

Most insights and actions were 'Fixes' (203 Fix (55\%), 154 Improve (42\%), 11 Change (3\%) occurring between 8 April and 1 May 2020), reflecting the short operational time of the hospital (treating 54 patients over 6 weeks). The proportion of fixes was expected to reduce over time as the system stabilised.

BLCs were encouraged to listen and support, rather than tell: the BLC was intended neither as an inspection nor educational role. Moreover, the value of the BLC role would be limited if the insights they reported did not lead to rapid change. Addressing 'fixes' quickly would ensure that staff's input had immediate, visible impact. Informal staff feedback about the BLC role was positive-things they cared about improved and made them feel listened to- and increased their commitment to the hospitals' learning ethos. Staff were consequently motivated to invest time on a busy shift to interact with the BLC.

\section{NATURE AND RHYTHM OF GATHERING INSIGHTS}

Learning from experience within the delivery organisation has a different cadence to learning from research conducted outside of it. Insights can be generated at any time, day and night, in line with the hospital operational hours.

The duration and pattern of BLC shifts changed over time. Initially, there were three BLC shifts per day (07:0012:30, 12:30-18:00, 18:00-00:00), 7 days/week, which generated high volumes of actionable insights in the early weeks and enabled observation of staff shift handovers. As the system stabilised, it was felt that fewer shifts were required. At the time of the hospital's hibernation (where the hospital was closed but remained in a state of readiness to reopen at 72 hours' notice if required) there were two BLC shifts per day (10:00-16:00, 18:00-00:00), 7 days/ week.

While reporting systems are designed for all incidents, whether they result in harm or not, reported incidents tend to focus on negative events. They seldom provide insights into what is working well and should be maintained or identify positive deviations (things which went unexpectedly well). As such, integrating BLC data with incident reporting can provide a more balanced understanding of the functioning of the ward.

A core group of 10, mostly clinicians (doctors, nurses and pharmacists), recruited to the 'BLC faculty', covered the majority of shifts. Most were working in the education faculty at the Nightingale, others were completing clinical shifts at other hospitals. The BLC role was designed to work alongside a lead clinician, in this case the matron, covering three wards of 42 beds each.

Other staff working at the hospital completed ad hoc BLC shifts. Staff feedback suggested this enhanced understanding of and commitment to building and maintaining a learning culture. Having a variety of professional backgrounds enhanced cross-disciplinary collaboration and enabled specialised input into problems. For example, after mouthcare was flagged as an area for improvement, a speech and language therapist completed a BLC shift to give specialist insights and recommendations. The ad hoc shifts included a wider range of clinicians (dieticians, speech and language therapists, physiotherapists, occupational therapists) and a range of seniorities (matrons, clinical directors, chief nurse, chief pharmacist). This diversity enhanced the nature of insights which benefited from multiple professional expertise and experiences.

\section{INTRODUCING BLCS INTO AN ORGANISATION}

It is unlikely that experience-based insights from front-line staff and patients would be implemented any quicker than those from clinical research without a specific mechanism to put those insights into action as fast as possible. The BLC provides an important data flow, but the effectiveness of the role requires additional system components to rapidly design, agree and deliver change. These include a coordinating function for the insights (in this case the Quality and Learning Team), organisational testing and evaluation capabilities, clinical and operational decision groups to respond and authorise changes swiftly, all connected into the learning cycle and reinforced by culture and behaviours. Therefore, we would caution against introducing the BLC role in isolation, without the wider learning system; doing so could risk 'listening' without 'acting', raising expectations among staff and patients which may not be met.

When adopting the role, a training and induction package is recommended to ensure BLCs are appropriately prepared for the unique positioning of the role to other staff and the unit. There are packages being used by units across the NHS that have begun to implement the role. These include observation skills, interview techniques, thematic data analysis and synthesis.

Patients arrived at the Nightingale already ventilated and without relatives accompanying them. While this was not done by BLCs at the Nightingale, we would recommend that those adopting the role include capture of patient, family and carer insights, which is well evidenced to lead to higher quality care.

The return on investment of the BLC has not been formally evaluated. The range and speed of insights captured and implemented responses, particularly for incidents, alongside wider staff benefits, underpinned leadership commitment to the role at the Nightingale. The diversity of BLCs' professional backgrounds and seniority was felt to enhance the quality of insights but meant there is no single pay band or job description for BLC shifts, which were paid activity.

Figure 2 outlines additional components suggested to maximise the value of the BLC role. 


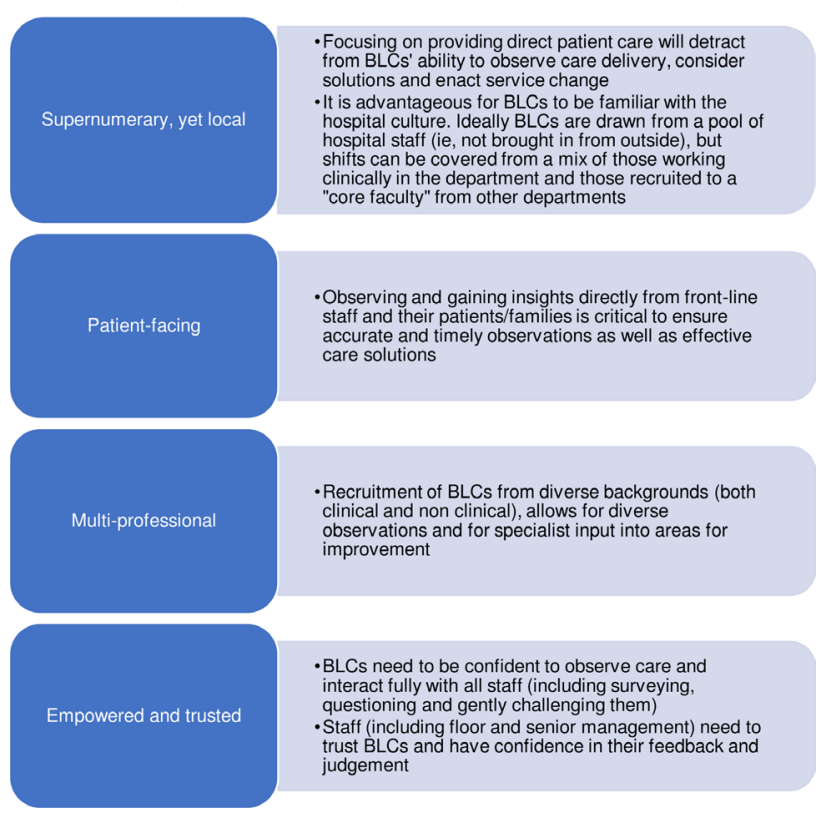

Figure 2 Maximising the value of the Bedside Learning Coordinator (BLC) role. Alongside operating within a broader learning system, we suggest that the above components are likely to be needed to get the full value from the BLC role.

\section{CONCLUSION}

Roles serving equivalent functions to the BLC have been used widely in manufacturing industries and other service settings outside the healthcare sector (eg, the Toyota Production System) to create systematic insights from front-line experience. We believe the BLC is a novel and valuable role throughout healthcare, not just in emergency field hospital settings, and can create valuable experienced-based data to inform change.

The BLC role at the Nightingale provided a mechanism to add additional internal data from front-line staff to be analysed alongside routine data to create richer insights for improvement. Investing in a mechanism to gather, act on and learn from staff, patient and relative insights can provide voice and agency for staff and deliver tangible improvement as part of a wider system of rapid, reliable learning. This mechanism can fundamentally change how we gather and act on front-line insights in a systematic way to deliver improvement.

\footnotetext{
Author affiliations

${ }^{1}$ UCLPartners, London, UK

${ }^{2}$ Department of Applied Health Research, University College London, London, UK

${ }^{3}$ Medical Directors Office, Imperial College Healthcare NHS Trust, London, UK ${ }^{4}$ Improvement Directorate, The Health Foundation, London, UK

${ }^{5}$ Burns Unit, Queen Victoria Hospital NHS Foundation Trust, East Grinstead, UK ${ }^{6}$ Department of Anaesthesia, The Royal National Orthopaedic Hospital NHS Trust, Stanmore, UK

${ }^{7}$ Department of Adult Nursing, King's College London, London, UK

${ }^{8}$ Transplant, Renal and Urology, Guy's and St Thomas' Hospitals NHS Trust, London, UK
}

${ }^{9}$ Trauma and Orthopaedic Surgery, Barking Havering and Redbridge Hospitals NHS Trust, Romford, UK

${ }^{10}$ Department of Anaesthesia, Royal Free London NHS Foundation Trust, London, UK

${ }^{11}$ Queen Alexandra Royal Army Nursing Corps, British Army, Camberley, UK

${ }^{12}$ Defence Primary Healthcare, Colchester Group Practice, Colchester, UK

${ }^{13}$ Medical Directorate, Royal Free London NHS Foundation Trust, London, UK

${ }^{14}$ Nuffield Trust, London, UK

Twitter Jenny Shand @JenShand_, Dominique Allwood $@$ DrDominiqueAllw, Karen Chui @Karenkchui and James Mountford@mountfordjames

Acknowledgements The authors thank the staff at NHS Nightingale Hospital London for their participation in the learning health system and for their support for the Bedside Learning Coordinator. Particular thanks to those who did Bedside Learning Coordinator shifts and to the leadership team who were instrumental in creating the conditions for success.

Contributors JS, DA, RB and JM designed and implemented the BLC programme at NHS Nightingale Hospital London. All authors participated in the BLC programme at NHS Nightingale Hospital London. JS wrote the first draft of the article. All authors reviewed and edited the drafts of the article. $\mathrm{RB}$ provided oversight and supervision throughout.

Funding The authors have not declared a specific grant for this research from any funding agency in the public, commercial or not-for-profit sectors.

Competing interests None declared.

\section{Patient consent for publication Not required.}

Provenance and peer review Not commissioned; externally peer reviewed.

Open access This is an open access article distributed in accordance with the Creative Commons Attribution Non Commercial (CC BY-NC 4.0) license, which permits others to distribute, remix, adapt, build upon this work noncommercially, and license their derivative works on different terms, provided the original work is properly cited, appropriate credit is given, any changes made indicated, and the use is noncommercial. See: http://creativecommons.org/licenses/by-nc/4. $0 /$.

ORCID iD

Jenny Shand http://orcid.org/0000-0002-4899-795X

\section{REFERENCES}

1 Bohmer R, Shand J, Bohmer R, et al. Learning systems: managing uncertainty in the new normal of Covid-19. NEJM Catal Innov Care Deliv 2020.

2 Tucker AL, Edmondson AC, Spear S. When problem solving prevents organizational learning. Journal of OrgChange Mgmt 2002;15:122-37.

3 Bohn R. Stop fighting the fires. Harvard Business Review 2000;78:83-92.

4 Green LW, Ottoson JM, García C, et al. Diffusion theory and knowledge Dissemination, utilization, and integration in public health. Annu Rev Public Health 2009;30:151-74.

5 Marshall M, Pagel C, French C, et al. Moving improvement research closer to practice: the Researcher-in-Residence model. BMJ Qual Saf 2014;23:801-5.

6 Lohr KN, Kathleen N. Rating the strength of scientific evidence: relevance for quality improvement programs. Int J Qual Health Care 2004;16:9-18.

7 Batalden M, Batalden P, Margolis P, et al. Coproduction of healthcare service. BMJ Qual Saf 2016;25:509-17. 